\title{
Layer-By-Layer Assembled, Amphiphilic And Antibacterial Hybrid Electrospun Mat Made From Polypropylene And Chitosan Fibers
}

Gokhan Acik ( $\nabla$ gacik@pirireis.edu.tr)

Piri Reis University https://orcid.org/0000-0002-9427-0508

\section{Burcu Acik}

Yalova Üniversitesi Merkez Yerleşkesi: Yalova Universitesi

E. Agel

TUBITAK Marmara Research Centre: Tubitak Marmara Arastirma Merkezi

\section{Research Article}

Keywords: amphiphilic, antibacterial, chitosan, chlorinated polypropylene, electrospinning, fiber mat

Posted Date: August 18th, 2021

DOl: https://doi.org/10.21203/rs.3.rs-796074/v1

License: (1) (i) This work is licensed under a Creative Commons Attribution 4.0 International License.

Read Full License

Version of Record: A version of this preprint was published at Journal of Polymers and the Environment on November 8th, 2021. See the published version at https://doi.org/10.1007/s10924-021-02324-x. 


\section{Abstract}

In this study, hybrid fiber mat (HFPP-CS) consisting of both chlorinated polypropylene and chitosan fibers (FPP-Cl and FCS) is obtained by assembling layer-by-layer (LBL) for the first time using electrospinning process. Morphological, wettability, structural and thermal properties of HFPP-CS are investigated in detail by Scanning electron microscopy (SEM), water contact angle (WCA) measurement, fourier transform infrared (FT-IR) spectroscopy, thermogravimetric analysis (TGA) and differential scanning calorimetry (DSC) analyses, respectively, comparing with FPP-Cl and FCS. Furthermore, the antibacterial activity of all samples was evaluated against to gram positive Staphylococcus aureus (S. aureus) and gram negative Escherichia coli $(E$. coli) bacteria. SEM analysis proves to HFPP-CS has a circular and smooth morphology and also comprises of microscale FPP-Cl and nanoscale FCS layers. The incorporation of nano-FCS layer on micro-FPP-Cl layer leads to diminution in the hydrophobicity, but enhancement in the thermostability and glass transition temperature of the resulting fiber mat. The antibacterial activity results show that HFPP-CS has inhibition effect against to $S$. aureus and $E$. coli. In general, it is anticipated that the prepared amphiphilic and antibacterial HFPP-CS can be employed as potential biomaterial for a variety of bioengineering applications.

\section{Introduction}

Polyolefins (POs), in general consisting of only hydrogen and carbon atoms, are widely utilized in a variety of emerging commodity applications covering from plastics, elastomers to fibers, due to their advantages of superior thermal and environmental stability, easy processability, high recyclability, corrosion and chemical resistance [1, 2]. In this context, they account for a very large part of the annual production capacity of the global plastic industry [3]. However, besides their many unique features, they represent some drawbacks that limit their usage, including low reactivity, poor adhesion, high photo oxidation sensitivity and incompatibility with other materials $[4,5]$. Thus, the modification of POs without sacrificing their service performance has long been a highly desirable subject of study for both industrial and academic researchers [6, 7]. Halogenation process [8], copolymerization [9], grafting [10], plasma treatment [11], impregnation [12], blending [13] and nanoparticle introducing [14] are among the reasonable addressed ways in the literature to extend the range of their applications. The introduction of functional groups by halogenation enhances the adhesion, solvent resistance, and gas permeability but also provide reactive sides which can be used in several well-known chemical reactions of the olefin backbone $[15,16]$. Among the halogenated POs, chlorinated polypropylene (PP-Cl) has several unique features such as heat and light stability, and resistance to abrasion, age, acid and alkali [17] and has also been extensively evaluated in many research studies for different purposes [18-22].

Chitosan (CS), as a kind of polysaccharide biopolymer, has distinct properties such as biodegradability, nontoxicity, low immunogenicity, good biocompatibility, antibacterial activity and film forming capacity, making it an interesting compound for bio-applications [23]. The combination of these many special properties with those of the synthetic polymers that build up the backbone is widely required in biomaterial design and development. Therefore, researchers have proposed the combined use of 
synthetic and natural polymers in recent years. However, the reported studies require the more compelling, harsh and restrictive workup conditions which cause safety issues [24]. In this regard, the layer-by-layer (LBL) assembly technique is regarded as a low-cost, simple, reliable and effective technique for producing uniform films through sequential deposition of species via secondary or electrostatic interactions, or hydrogen bonding [25].

Among the fiber production techniques [26], electrospinning providing the high surface area to volume ratio, is utilized to fabricate the fibers with well-controlled surface topography, morphology and size distribution at micro- or nano-scale range. This method, which can be applied to both natural and synthetic polymers and inorganic materials when appropriate conditions are provided, is a versatile for many applications particularly like sensor technology, textile, filtration, protective clothing, wound dressing, drug delivery, etc [27-32]. It is clearly understood that the LBL deposition of different fibers made from different polymers by electro-spinning is an effective process that will be able to utilize to produce composite multilayer scaffolds.

Based on the above information, hybrid fiber mat possessing FPP-Cl and FCS were developed by LBL technique using electrospinning. The concerned properties of resultant composite fibrous mat and its precursors were investigated accordingly in terms of morphology, wettability and thermal properties. The main focus was to find out to what extent PP-Cl / CS bicomponent fiber mats will exhibit antibacterial activity.

\section{Experimental}

\subsection{Materials}

Commercially chlorinated polypropylene (PP-Cl, chlorine mass fraction: $29-32 \%[\mathrm{~m} / \mathrm{m}]$ quoted from product catalogue, $M_{\mathrm{n}, \mathrm{GPC}}=50,000 \mathrm{~g} \cdot \mathrm{mol}^{-1}$ determined by GPC measurement) was purchased from Mark Zhang Shanghai Sunking Industry Incorporation (Shanghai, China). Medium molecular weight chitosan (CS, molecular weight of $190-310 \mathrm{kDa}$, deacetylation degree of $75-85 \%, 200-800 \mathrm{cP}, 1 \mathrm{wt}$. \% in $1 \%$ acetic acid at $25^{\circ} \mathrm{C}$ ) was procured from Sigma Aldrich (Steinheim, Germany). The solvents utilized to prepare the polymer solutions such as, tetrahydrofuran (THF, $\geq 99.5 \%$ ), dichloromethane (DCM, $\geq 99.5 \%$ ) and trifluoroacetic acid (TFA, $\geq 99.0 \%$ ) were supplied from Sigma Aldrich (Steinheim, Germany). Precleaned microscope glass slides ( $3 \times 1$ inch) were supplied from ISOLAB (Istanbul, Turkey). The bacterial strains used in this study include Gram negative bacteria, Escherichia coli (ATCC 25922), and Gram positive bacteria, Staphylococcus aureus (ATCC 29213). S. aureus and E. coli were inoculated into Muller Hinton Agar (Merck, Darmstadt, Germany) medium for general production purposes.

\subsection{Instrumentation}

The attenuated total reflectance Fourier transform infrared (ATR-FTIR) spectra of the samples were recorded by Perkin-Elmer Spectrum BX spectrometer over a range of wavenumber $4000-400 \mathrm{~cm}^{-1}$ with 4 $\mathrm{cm}^{-1}$ resolution and by averaging 16 scans. Water wettability of surfaces of the specimens was 
determined on a Theta Optical Tensiometer device (CAM-200) and water droplet photographs were captured with a conventional digital camera. The other surface properties such as topography and morphology of the samples were examined by Scanning electron microscope (SEM, JEOL JSM 6510-LV, IET International Equipment Trading Ltd. Mundelein, Illinois USA) observations. The samples were coated with palladium using Polaron Sputter Coater SC7640 (Quorum Technologies, East Sussex, UK) to be examined by means of SEM. Thermal stabilities of the samples were determined by conducting thermogravimetric analysis (TGA, SeikoSII TG-DTA 6300 TG/DTA analyzer) under nitrogen atmosphere at the scanning range of $25-600^{\circ} \mathrm{C}$ and heating rate of $10^{\circ} \mathrm{C} / \mathrm{min}$. The glass transition temperatures of the samples were determined by differential scanning calorimetry (DSC, Mettler Toledo DSC analyzer) analysis at the scanning range of 25 to $100^{\circ} \mathrm{C}$ and 25 to $400^{\circ} \mathrm{C}$ by $10^{\circ} \mathrm{C} / \mathrm{min}$ heating rate using a nitrogen flow. The samples were weighed approximately to $3-5 \mathrm{mg}$ before the both thermal analyses.

\subsection{Fabrication of electrospun mat made of polypropylene and chitosan fibers (HFPP-CS)}

The layer-by-layer ( $\mathrm{LBL}$ ) assembly technique was used to fabricate the amphiphilic HFPP-CS and its precursor fibers by means of electrospinning. Firstly, pure PP-Cl electrospinning solution $(20 \%, \mathrm{w} / \mathrm{w})$ was prepared in THF at room temperature under constant stirring for three hours until a homogeneous solution was achieved. Then, the microfibers of PP-Cl were fabricated by electrospinning by using parameters of tip-to-collector distance (TCD) of $17 \mathrm{~cm}, 15 \mathrm{kV}$ applied voltage and $1.3 \mathrm{~mL}^{-1} \mathrm{~h}^{-1}$ flow rate. On the other hand, pristine CS electrospinning solution $(5 \%, \mathrm{w} / \mathrm{w})$ prepared in TFA and DCM $(7: 3, \mathrm{v} / \mathrm{v})$ solvent mixture at room temperature for overnight. The TCD, applied voltage and flow rate was kept at $10 \mathrm{~cm}, 15$ $\mathrm{kV}$ and $0.1 \mathrm{~mL} . \mathrm{h}^{-1}$, respectively to achieve the nanofibers of CS. The HFPP-CS composite mat was prepared by electrospinning from the pristine PP-Cl firstly, and then CS was electrospun onto the surface of as-spun PP-Cl fibers. In all cases, a typical laboratory scale NE-100 model electrospinning set up (Inovenso, Turkey) was utilized. The solutions were poured into a plastic syringe $(5 \mathrm{~mL})$ with a metal needle tip which was driven by a syringe pump (New Era Pump Systems Inc.) and a high voltage power supply (Elektrosis, PW1010, Turkey). Moreover, electrospinning durations were $1 \mathrm{~h}$ and $30 \mathrm{~min}$ for PP-Cl and CS, respectively. During the electrospinning processes, laboratory temperature was $23.1^{\circ} \mathrm{C}$, whereas relative humidity was $50 \%$.

\subsection{Water contact angle measurements}

The static water contact angle (WCA) of the achieved composite fiber mat and its pristine precursors coated on microscope glass slides was analyzed by placing $5 \mu \mathrm{L}$ of ultra-pure water onto sample surfaces by a computer-controlled liquid dispensing system. The system consists of a syringe pump, a micro-syringe equipped with stainless steel needle. A conventional digital camera was utilized to capture the representative images of water droplet on the sample surfaces. The average of left and right contact angles was reported after 8 measurements on different places of the specimens with \pm SD by using Laplace-Young fitting algorithm.

\subsection{In vitro bactericidal efficacy test}


The lowest antimicrobial agent concentration at which a microorganism is visibly inhibited from growth is described as the "Minimal Inhibitory Concentration (MIC)". Dilution methods are used to determine the MIC values of antimicrobial agents. Microdilution test; It is widely preferred in the MIC determination of compounds with potential antimicrobial activity due to advantages such as sensitivity, reproducibility, and ease of application. In this work, microdilution test was performed according to CLSI (Clinical and Laboratory Standards Institute) instructions.

\subsubsection{Bacterial suspension preparation procedure}

E. coli (ATCC 25922) and S. aureus (ATCC 29213) were inoculated into Muller Hinton Agar medium and incubated for 18-24 hours. At the end of the incubation period, a single colony was selected from the plate and the density of the bacterial suspension homogenized by vortex agitation was adjusted by reading the $0.5 \mathrm{McF}$ arland turbidity $\left(1 \times 10^{8} \mathrm{CFU} / \mathrm{ml}\right)$ standard with physiological sterile saline solution $(0.9 \% \mathrm{NaCl})$ using a densitometer (Densimat; Biomé rieux, Marcy l'Etoile, France). The bacterial suspension $\left(1 \times 10^{8} \mathrm{CFU} / \mathrm{ml}\right)$ adjusted to $0.5 \mathrm{McF}$ arland was diluted $1 / 10$ to obtain $10^{7} \mathrm{CFU} / \mathrm{ml} .5 \mu \mathrm{L}$ final bacteria suspension was pipetted into microplate wells for each cases. Schematic representation for preparation of bacterial suspensions was illustrated in Fig. 1.

\subsubsection{Application of the microdilution test}

96-well U-bottom sterile Tissue Culture Testplate microplate (TPP, Switzerland) was used for the test. 100 $\mu \mathrm{L}$ of cation-adjusted sterile BBL Müller Hinton Broth II (CAMHB, Becton Dickinson, USA) was pipetted under aseptic conditions into all wells of the microplate. The entire surface of microscope glass slides covered with electrospun fibers was scraped and $1 \mu \mathrm{g}$ was taken. $1 \mu \mathrm{g}$ of electrospun fibers were homogenized in $1 \mathrm{~mL}$ CAMHB and $100 \mu \mathrm{L}$ was pipetted into the first well. Serial dilution was made by taking $100 \mu \mathrm{L}$ from this first well and transferring it to the other well. Dilution was continued until well number 8 and $100 \mu \mathrm{L}$ was ejected from this well. Wells numbered 9-10 were arranged as sterility control (SC) and well number 11 and 12 were labelled as E.coli and S.aureus negative bacteria growth control well (NC), respectively. From the standardized bacterial suspension of $10^{7} \mathrm{CFU} / \mathrm{ml}, 5 \mu \mathrm{l}$ was pipetted into all wells except sterility control wells numbered 9-10.

\section{Results And Discussion}

The preparation of CS based materials has received growing attention in extensive biomedical fields due to its inherent properties particularly including nontoxicity, antibacterial activity, biocompatibility and biodegradability [33]. Especially, it is well known that the antibacterial activity of chitosan is high against a wide variety of fungi, yeast, bacteria and viruses [34]. Meanwhile, the electrospinning of chitosan is hard to achieve due to the complicated behavior of its solution. In this regard, some polymers such as PLA [35], PVA [36] or PEO [37] were used to improve electrospinnability of chitosan. Furthermore, it was proven by the studies in the literature that the smooth and bead-free nanofibers of pristine CS were attained from its solution in TFA/DCM cosolvent system [38,39]. On the other hand, the electrospun 
microfibers of PP-Cl are achieved under ambient conditions in our laboratory for the first time in the literature [21]. However, to the best of our knowledge, there is no report on evaluating the LBL assembled PP-Cl and CS fibers based hybrid mat fabricated by electrospinning and its antibacterial properties. Advances in the modification of composite fibers produced by the LBL assembly technique in solutions and in combining the properties of natural and synthetic polymers gave us the idea for the production of PP-Cl and CS based fibrous mats for biomedical applications. In this purpose, firstly, the fabricated electrospun FPP-Cl, FCS and HFPP-CS were morphologically compared by using SEM analysis (Fig. 2). As illustrated in Fig. 2, pristine FPP-Cl and FCS exhibited nearly uniform fiber morphology and cylindrical fibers within the range of 2.1-2.7 $\mu \mathrm{m}$ and $175-475 \mathrm{~nm}$, respectively. Moreover, the comparison between precursor micro and nanofibers and HFPP-CS fibers, it was worth noting that CS nanofibers were successfully adhered onto PP-Cl microfibers by LBL deposition. The supporting information about this finding was the probable intermolecular electrostatic interactions between chlorine functionalities of PP$\mathrm{Cl}$ and CS main chain [40].

LBL assembly can be used as an effective one-step surface modification technique to alter the wettability of electrospun mats. Two alternative routes are possible so as to achieve amphiphilization of electrospun mats: (i) preparation of electrospinning solution by mixing the hydrophobic and hydrophilic or amphiphilic polymer and (ii) LBL deposition of these polymers on each other during the electrospinning. In our study using the second approach, the first layer was hydrophobic PP-Cl microfibers, whereas the second layer was hydrophilic CS nanofibers. Sessile drop technique was used for investigating the resulting hydrophilic-hydrophobic transitions. After the assembly of a hydrophobic and a hydrophilic polymer by the LBL technique using electrospinning, a WCA value of $124^{\circ} \pm 2$ determined for HFPP-CS which was between the WCA of both polymers proved that the HFPP-CS obtained was amphiphilic, as expected (Fig. 3).

The LBL assembling of electrospun FPP-Cl and FCS was structurally elucidated by observing specific group peaks by comparative FT-IR spectroscopy study, as shown in Fig. 4. First, it was found that the absorption observed around 2940 and $735 \mathrm{~cm}^{-1}$ corresponding to the asymmetric and symmetric stretching vibrations of $\mathrm{C}-\mathrm{H}$ and stretching vibrations of $\mathrm{C}-\mathrm{Cl}$ groups of electrospun $\mathrm{FPP}-\mathrm{Cl}$, respectively [16]. The specific signals, including the $-\mathrm{OH}$ stretching at $3450 \mathrm{~cm}^{-1}, \mathrm{C}-\mathrm{H}$ stretching at $2900 \mathrm{~cm}^{-1}$, bending of amide I at $1670 \mathrm{~cm}^{-1}$, amide II at $1520 \mathrm{~cm}^{-1}$ and amide III at $1420 \mathrm{~cm}^{-1}, \mathrm{C}-\mathrm{O}$ stretching vibrations at 1370 and $1030 \mathrm{~cm}^{-1}$ were clearly observed for electrospun FCS[40]. After the LBL assembling by electrospinning, the intensified $-\mathrm{OH}$, amide I, II and III stretching vibrations of HFPP-CS compared to pristine FCS clearly confirmed the presence of the intermolecular electrostatic interactions between PP-Cl and CS main chains [40]. In addition, the other important finding was the preservation of all the major characteristic signals of both polymers in the HFPP-CS spectrum.

Evaluating the thermal features of electrospun mats consisting of different species is an important consideration, especially in the fields of biomedical applications. In this context, thermal stabilities and glass transition temperatures $\left(T_{\mathrm{g}} \mathrm{s}\right)$ of both precursor FPP-Cl and FCS as well as their LBL assembled mat 
were determined and comparatively studied by TGA and DSC analyses (Figs. 5 and 6). TGA was conducted to present the decomposition pattern and hence the compositional differences of the specimens at the scanning range of $25-600^{\circ} \mathrm{C}$. Thermogravimetric curves of pristine and LBL assembled electrospun were illustrated in Fig. 5. As seen in this figure, the exhibited two thermal maximum decomposition stages $\left(T_{1}\right.$ and $\left.T_{2}\right)$ recorded at $215^{\circ} \mathrm{C}$ and $350^{\circ} \mathrm{C}$ for FPP-Cl due to the dehydrochlorination and degradation of $\mathrm{PP}-\mathrm{Cl}$ main chain, while the same number of decomposition stages represented at around 100 and $200-500{ }^{\circ} \mathrm{C}$ was determined for FCS resulting from evaporation of the adsorbed water and degradation of polymeric CS backbone, respectively, in accordance with previously published results $[41,42]$. TGA curves also illustrated that the maximum degradation temperatures slightly shifted to higher for electrospun HFPP-CS specimen after LBL assembling of the precursors (Table 1). This result may be another indication of the presence of electrostatic interactions between the FPP-Cl and FCS. It was also seen from the DSC curves that LBL assembling positively affected the $T_{\mathrm{g}}$ of the resulting mat. As can be seen in Fig. $6, T_{\mathrm{g}}$ value of micro FPP-Cl was $45^{\circ} \mathrm{C}$, after the nano-FCS deposition on it, the $T_{\mathrm{g}}$ value enhanced to higher temperature due to the restrictive effect of hard CS rings on segmental mobility achieved mat or its more oriented chains [43]. Meanwhile, the endothermic and exothermic peaks of FCS curve determined at approximately between $80-120^{\circ} \mathrm{C}$ and $275-300{ }^{\circ} \mathrm{C}$ were corresponded to the evaporation of residual solvent and decomposition of chitosan backbone [44, 45]. All achieved results from the thermal analyses were also summarized in Table 1.

Table 1. Thermal and mechanical properties of the samples.

\begin{tabular}{lllll} 
Sample & $T_{1}{ }^{\mathrm{a}}$ & $T_{2}{ }^{\mathrm{a}}$ & $T_{\mathrm{g}}^{\mathrm{b}}$ & WCA $^{\mathrm{c}}$ \\
& $\left({ }^{\circ} \mathrm{C}\right)$ & $\left({ }^{\circ} \mathrm{C}\right)$ & $\left({ }^{\circ} \mathrm{C}\right)$ & $\left({ }^{\circ}\right)$ \\
\hline FPP-Cl & 215 & 325 & 40 & $137 \pm 2$ \\
\hline FCS & 190 & 350 & - & $82 \pm 2$ \\
\hline HFPP-CS & 305 & 340 & 48 & $124 \pm 2$
\end{tabular}

${ }^{a}$ The first and second degradation temperatures determined by TGA analysis.

${ }^{\mathrm{b}}$ Glass transition temperature determined by DSC analysis.

${ }^{c}$ Water contact angle determined by WCA analysis

The different nanotechnologies providing the large surface area have increasingly been utilized in order to develop the antibacterial materials especially for biomedical applications [46]. The electrospinning method facilitates the production of antibacterial fiber composites through the layered of neat polymeric fiber components from a solution or melt [47]. The antibacterial properties of HFPP-CS developed on the basis of these cases were assessed by determining MIC via broth dilution method (Fig. 7). After pipetting 
as described in application of the microdilution test section, the microplate was covered with adhesive foil to prevent drying and incubated at $37^{\circ} \mathrm{C}$ for $16-20$ hours. The lowest concentration of fibers without visible turbidity, which prevents the growth of bacteria, was evaluated as the minimum inhibitory concentration. It was clearly seen from the Fig. 7 that HFPP-CS had a lower than FPP-Cl, but higher MIC than FCS against both bacteria. These findings were as anticipated when the results from the WCA and SEM analyses were considered [26, 48-50].

\section{Conclusions}

Materials that are susceptible to bacterial invasion and infections compel researchers to develop various properties of these materials with innovative methods for many research areas. In the present research, a new dual-layer hybrid electrospun fiber mat possessing both synthetic and natural polymer with antibacterial activity was successfully fabricated. The lower layer included chlorinated polypropylene fibers surrounded by chitosan fibers as an external layer. SEM analysis proved that the lower FPP-Cl layer was thicker than FCS external layer. A significant effect of FCS deposition on the FPP-Cl on wettability, thermostability and $T_{\mathrm{g}}$ of the hybrid fiber mat was observed. Ultimately, the nano-FCS coated hybrid fibers had a good antibacterial activity against both gram positive and gram negative bacteria. Therefore, it is anticipated that the findings of presented study will provide new contributions to researchers particularly working on biomedical applications.

\section{Declarations}

ORCID

\section{G. Acik https://orcid.org/0000-0002-9427-0508}

\section{Funding}

This research did not receive any specific grant from funding agencies in the public, commercial, or notfor-profit sectors.

\section{Author contribution statement}

Gokhan Acik: Formal analysis, Investigation, Project administration, Data curation, Writing-original draft, Methodology, Conceptualization, Software, Supervision, Writing - Review \& Editing Burcu Acik: Formal analysis, Methodology, Investigation Esra Agel: Formal analysis, Antibacterial test, Investigation

\section{Declaration of Competing Interest}

The authors declare that they have no known competing financial interests or personal relationships that could have appeared to influence the work reported in this paper

\section{References}


1. Lopez RG, D’agosto F, Boisson C (2007) Prog Polym Sci 32:419

2. Chung $T$ (2002) Progress in polymer science 27:39

3. Sauter DW, Taoufik M, Boisson C (2017) Polymers 9:185

4. Chung TM (2013) Macromolecules 46:6671

5. Franssen NM, Reek JN, de Bruin B (2013) Chem Soc Rev 42:5809

6. Acik G, Sey E, Tasdelen M (2018) Express Polymer Letters 12:418

7. Arslan M, Acik G, Tasdelen MA (2019) Polymer Chemistry 10:3806

8. Plummer CM, Zhou H, Zhu W, Huang H, Liu L, Chen Y (2018) Polymer Chemistry 9:1309

9. Kay CJ, Goring PD, Burnett CA, Hornby B, Lewtas K, Morris S, Morton C, McNally T, Theaker GW, Waterson C (2018) J Am Chem Soc 140:13921

10. Acik G, Altinkok C, Tasdelen MA (2018) J Polym Sci Part A: Polym Chem 56:2595

11. Plummer CM, Li L, Chen $Y$ (2020) Polymer Chemistry $11: 6862$

12. Zhang X, Maira B, Hashimoto Y, Wada T, Chammingkwan P, Thakur A, Taniike T (2019) Composites Part B: Engineering 162:662

13. Agwuncha SC, Ibrahim ID, Sadiku ER (2017) Improving the thermal and flame resistance properties of polyolefins. I: Polyolefin Fibres, s. 421: Elsevier

14. Alonso YN, Grafia AL, Castillo LA, Barbosa SE (2021) Active Packaging Films Based on Polyolefins Modified by Organic and Inorganic Nanoparticles. I: Reactive and Functional Polymers Volume Three, s. 5: Springer

15. Mehmood A, Xu X, Kang X, Luo Y (2020) New J Chem 44:16941

16. Acik G, Tasdelen MA (2016) Polym Int 65:1458

17. Tang SY, Liu DZ, Fan ZL (2006) Phys Chem Liq 44:531

18. Acik G, Altinkok C, Olmez H, Tasdelen MA (2018) Prog Org Coat 125:73

19. Hazer B, Ashby RD (2021) Food Chem 344:128644

20. Bayramoğlu G, Hazer B, Altıntaş B, Arıca MY (2011) Process biochemistry 46:372

21. Acik G, Altinkok C (2019) J Appl Polym Sci 136:48199

22. Kalaycı ÖA, Cömert FB, Hazer B, Atalay T, Cavicchi KA, Cakmak M (2010) Polym Bull 65:215

23. Tao F, Ma S, Tao H, Jin L, Luo Y, Zheng J, Xiang W, Deng H (2020) Carbohydrate polymers 117063

24. Gozutok M, Basar AO, Sasmazel HT (2018) J Nanosci Nanotechnol 18:2881

25. Hammond PT (2012) Materials today 15:196

26. Daglar O, Altinkok C, Acik G, Durmaz H (2020) Macromol Chem Phys 221:2000310

27. Lu L, Yang B, Zhai Y, Liu J (2020) Nano Energy 76:104966

28. Lu Y, Xiao X, Fu J, Huan C, Qi S, Zhan Y, Zhu Y, Xu G (2019) Chem Eng J 355:532

29. Wang C, Fan J, Xu R, Zhang L, Zhong S, Wang W, Yu D (2019) Journal of Materials Science 54:12522

30. Baji A, Agarwal K, Oopath SV (2020) Polymers 12:492 
31. Ghosal K, Agatemor C, Špitálsky Z, Thomas S, Kny E (2019) Chem Eng J 358:1262

32. Ding Y, Li W, Zhang F, Liu Z, Zanjanizadeh Ezazi N, Liu D, Santos HA (2019) Adv Func Mater 29:1802852

33. Zhao D, Yu S, Sun B, Gao S, Guo S, Zhao K (2018) Polymers 10:462

34. Atay HY (2019) Antibacterial activity of chitosan-based systems. I: Functional chitosan, s. 457: Springer

35. Geng X, Kwon O-H, Jang J (2005) Biomaterials 26:5427

36. Gökhan A, Kamaci M, ÖZATA B, CANSOY CEÖ (2019) Turk J Chem 43:137

37. Duan B, Dong C, Yuan X, Yao K (2004) J Biomater Sci Polym Ed 15:797

38. Ohkawa K, Cha D, Kim H, Nishida A, Yamamoto H (2004) Macromol Rapid Commun 25:1600

39. Sangsanoh P, Supaphol P (2006) Biomacromol 7:2710

40. Kaya M, Khadem S, Cakmak YS, Mujtaba M, Ilk S, Akyuz L, Salaberria AM, Labidi J, Abdulqadir AH, Deligöz E (2018) RSC Adv 8:3941

41. Li W, Shi L, Shen D, Luo B (1988) Polym Degrad Stab 22:375

42. Abdelrazek E, Elashmawi I, Labeeb S (2010) Physica B: Condensed Matter 405:2021

43. Gupta A, Pal AK, Woo EM, Katiyar V (2018) Scientific reports 8:1

44. Koosha M, Mirzadeh H, Shokrgozar MA, Farokhi M (2015) RSC Adv 5:10479

45. Nair RS, Morris A, Billa N, Leong C-O (2019) AAPS PharmSciTech 20:69

46. Westmeier D, Hahlbrock A, Reinhardt C, Fröhlich-Nowoisky J, Wessler S, Vallet C, Pöschl U, Knauer S, Stauber R (2018) Chem Soc Rev 47:5312

47. Borjihan Q, Dong A (2020) Biomaterials science 8:6867

48. Gurol I, Altinkok C, Agel E, Tasaltin C, Durmuş M, Acik G (2020) J Coat Technol Res 17:1587

49. Altinkok C, Karabulut HF, Tasdelen MA, Acik G (2020) Materials Today Communications 25:101425

50. Gao Y, Bach Truong Y, Zhu Y, Louis Kyratzis I (2014) J Appl Polym Sci 131:40797

\section{Figures}

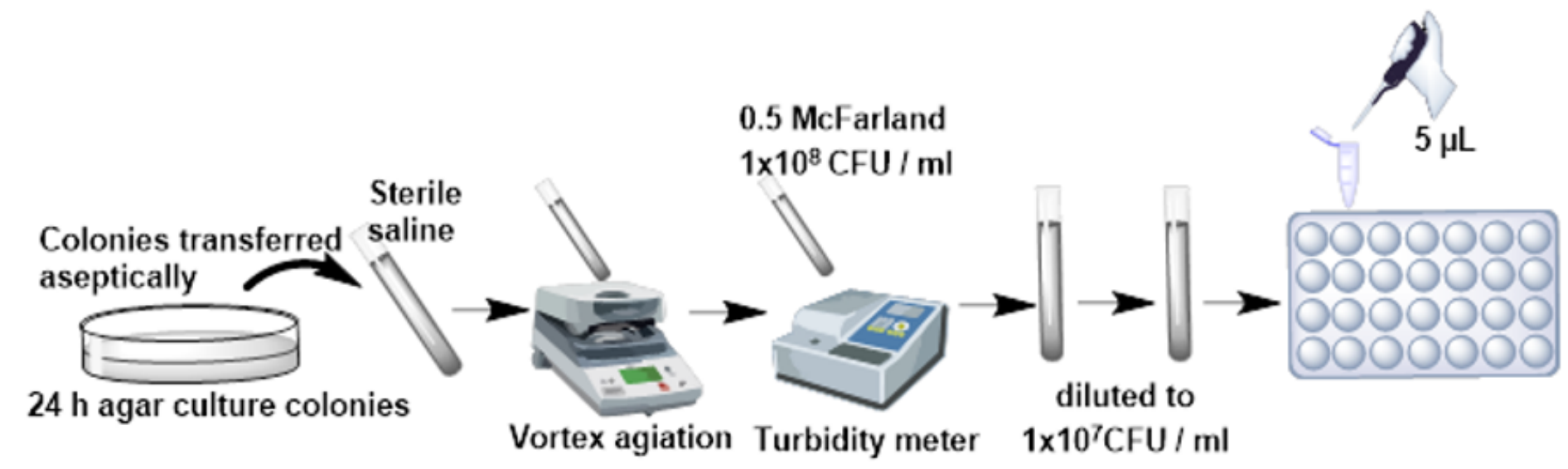




\section{Figure 1}

Overall preparation procedure for the bacterial suspension.

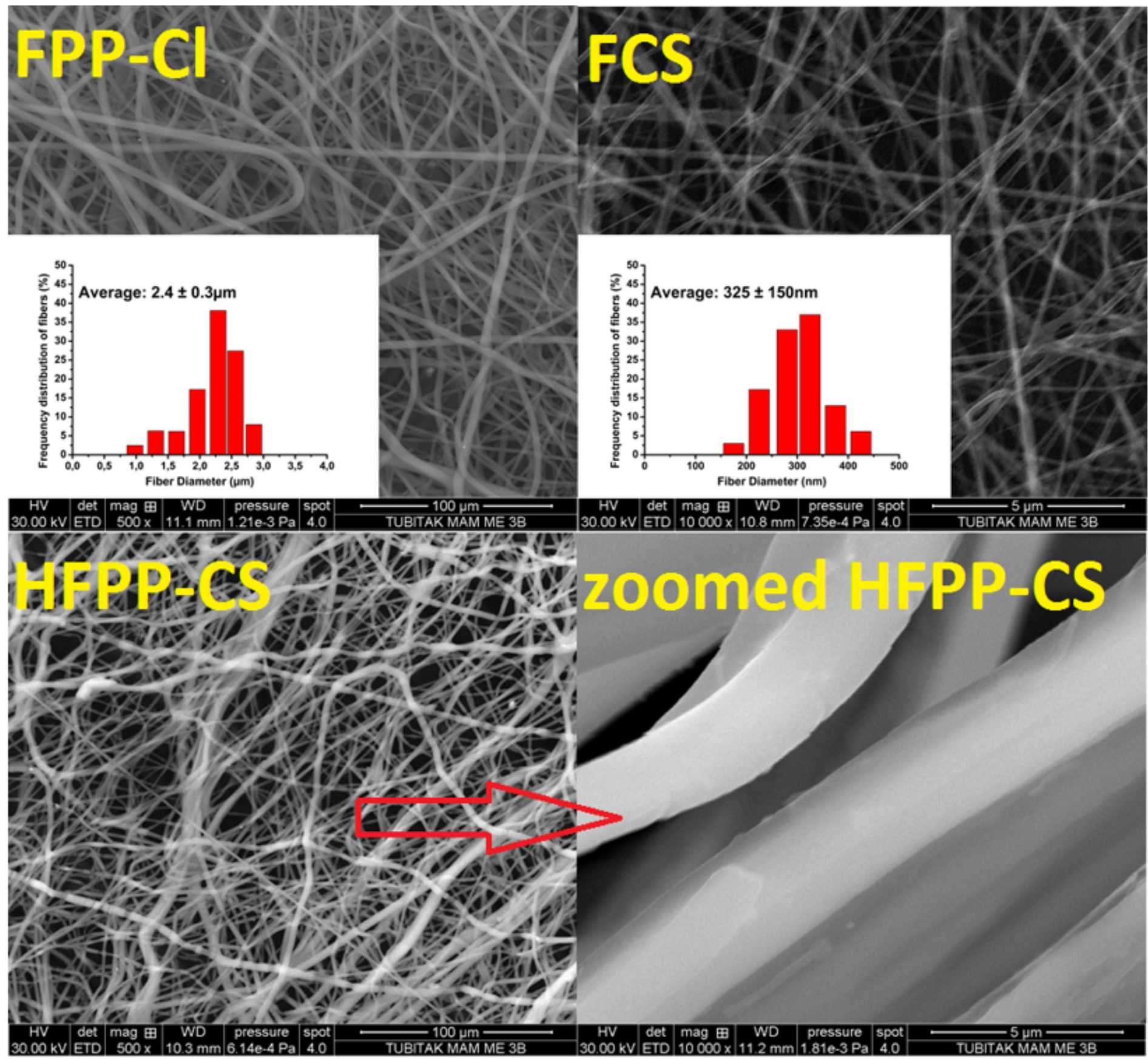

Figure 2

SEM images and diameter distribution histograms of FPP-Cl, FCS and their LBL assembled HFPP-CS. 


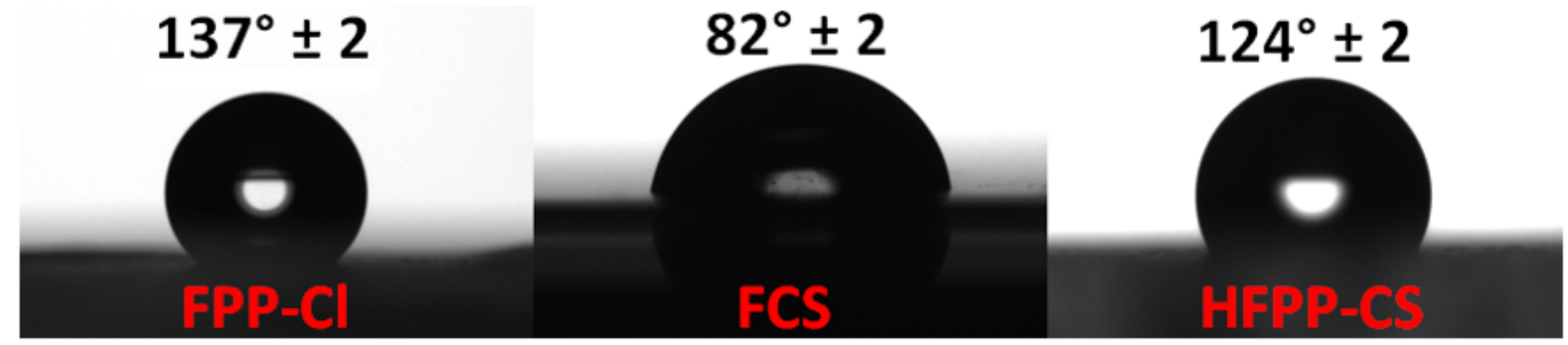

Figure 3

Optical images showing the static contact angle between sample surfaces and a droplet of deionized water.

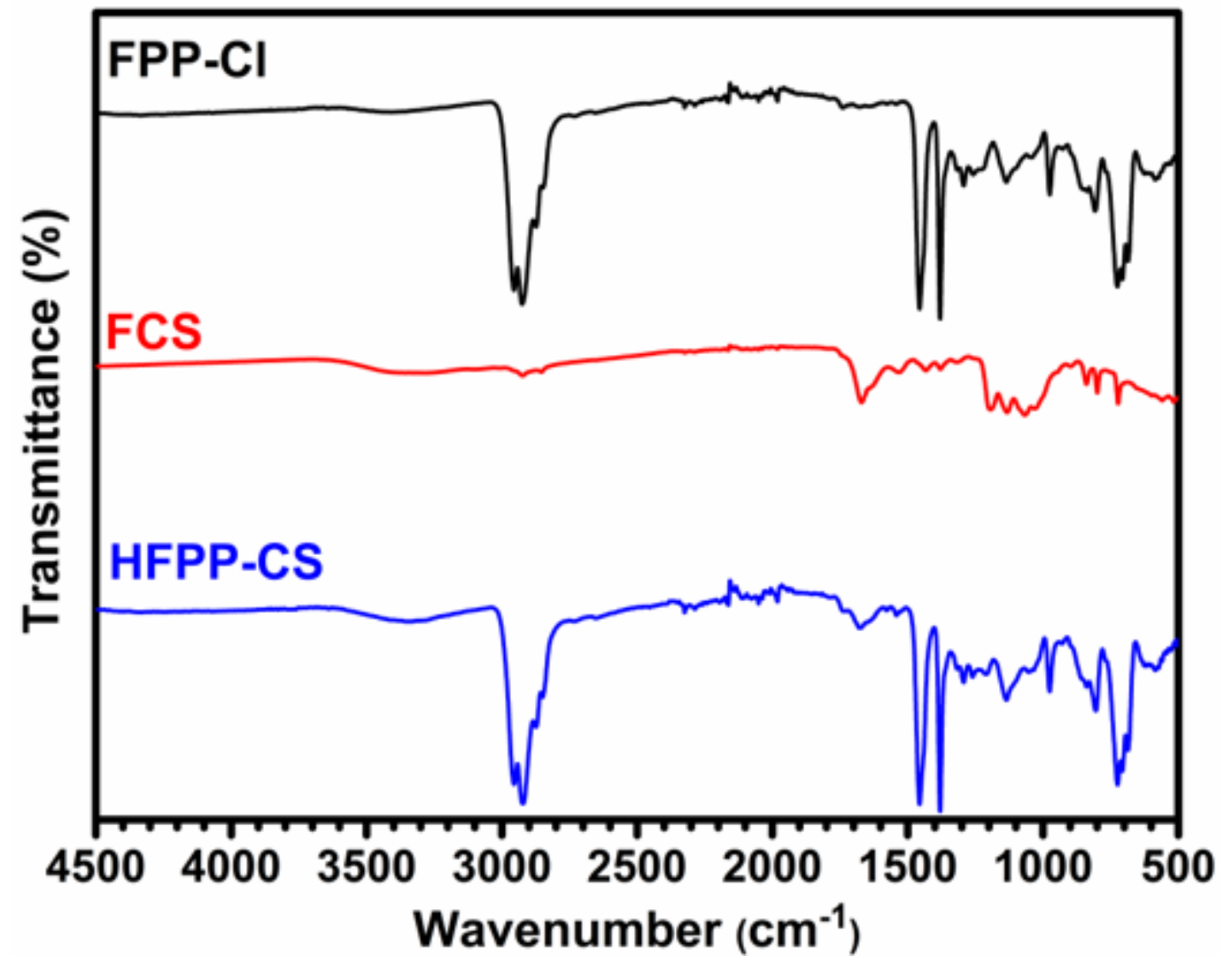

Figure 4

The ATR FT-IR spectra of electrospun FPP-Cl, FCS and HFPP-CS. 


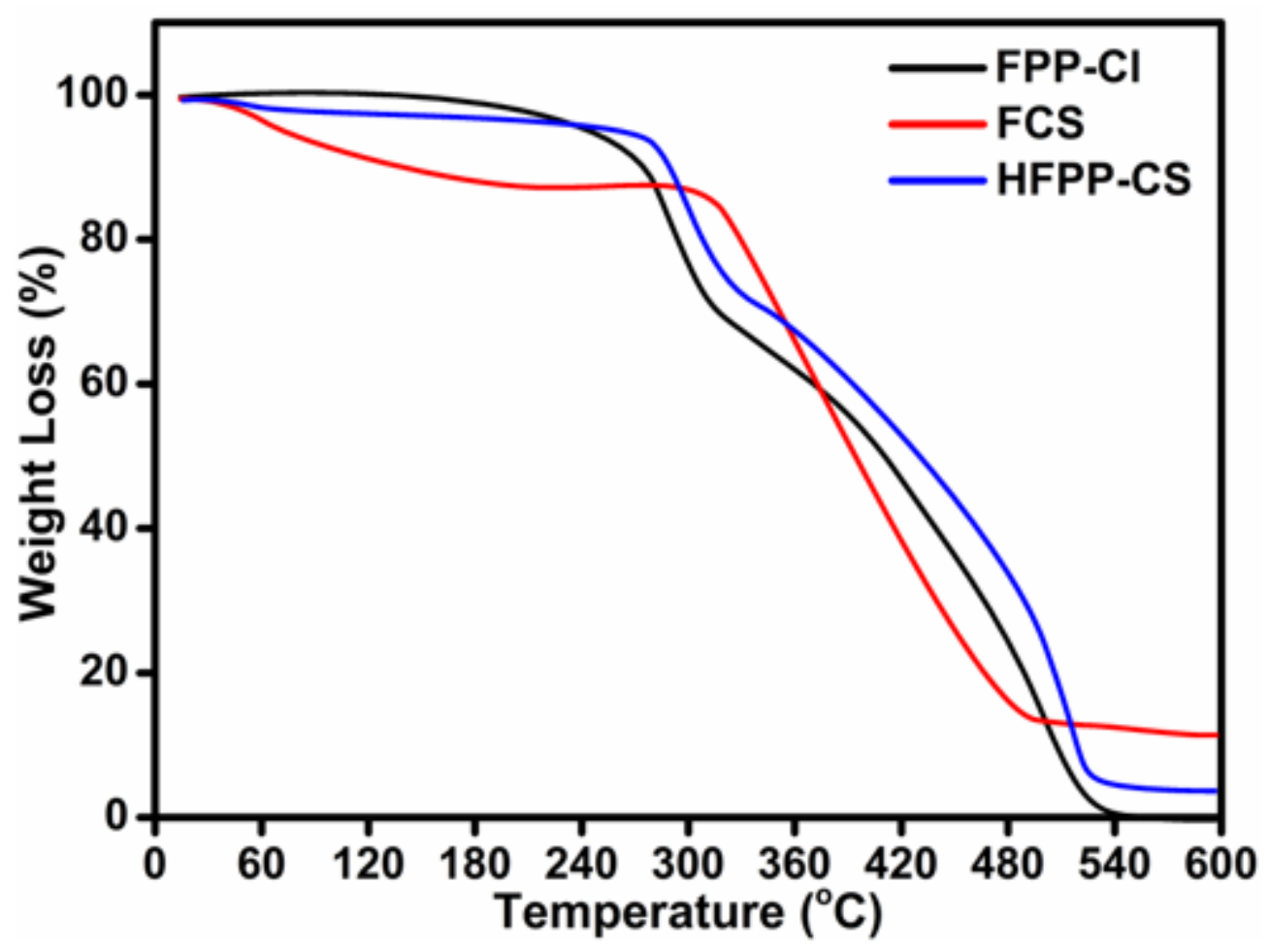

Figure 5

TGA curves of the electrospun HFPP-CS mat and its precursor fibers

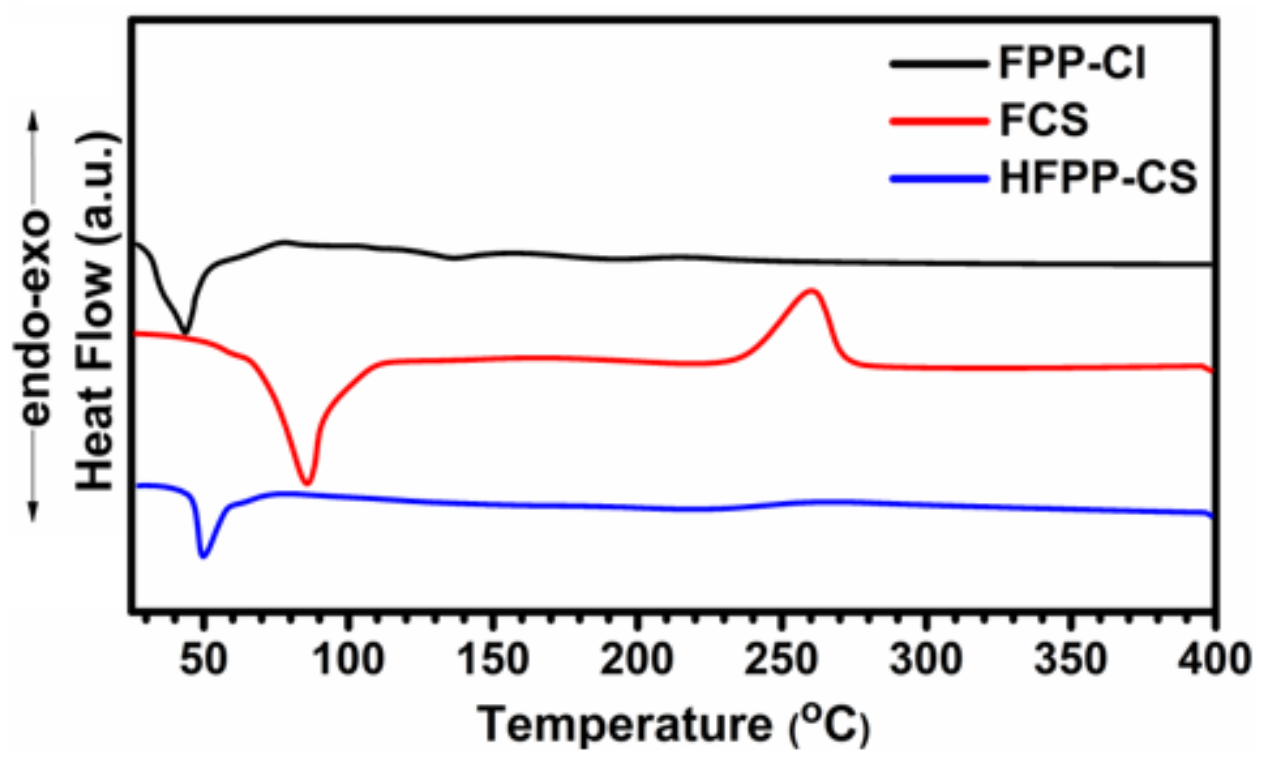

Figure 6

DSC thermograms of the electrospun HFPP-CS mat and its precursor fibers. 

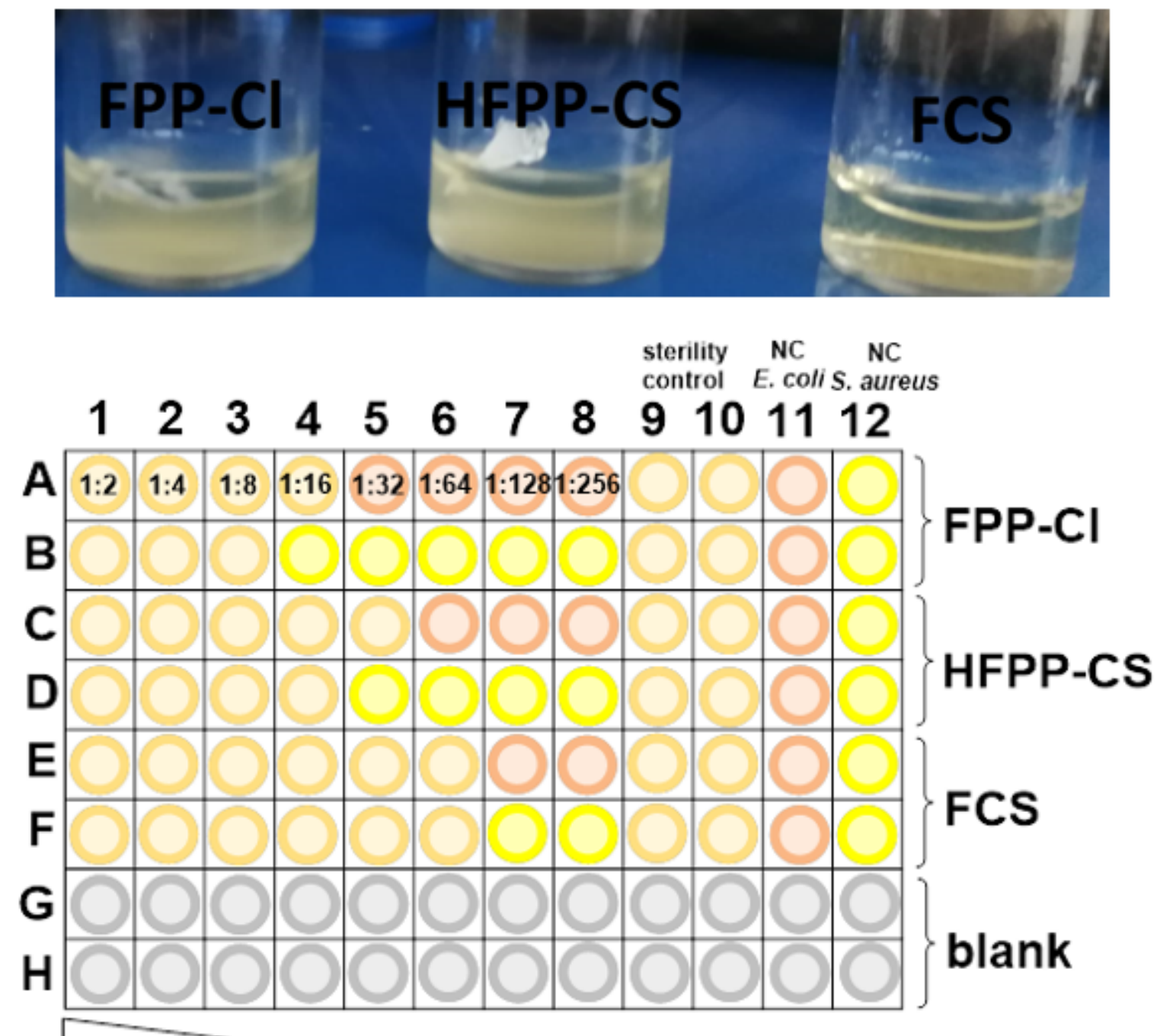

Concentration

S. aureus growth

E. coli growth

\section{no growth Blank}

Figure 7

Visual turbidity of samples in wells against to S. aureus (top) and schematic protocol for determination of growth-inhibitory effect of the samples in culture medium at which various dilutions (from 1:2 to 1:256) on S. aureus and E. coli (bottom).

\section{Supplementary Files}

This is a list of supplementary files associated with this preprint. Click to download.

- Graphicalabstract.docx 